\begin{tabular}{|c|l|}
\hline Title & Misorientation/ocal plastic strain manifestations in chemical etching color \\
\hline Author(s) & Zhang, X ianguang; Matsuura, Kiy otaka; Ohno, Munekazu \\
\hline Citation & $\begin{array}{l}\text { Micron, 59, 28-32 } \\
\text { https://loi.org/10.1016/.micron.2013.12.008 }\end{array}$ \\
\hline Issue Date & 201404 \\
\hline Doc URL & http://hdl.handle.net/2115/57599 \\
\hline Type & article (author version) \\
\hline File Information & 2013ZZhang-Orientation chemical etching-8M.pdf \\
\hline
\end{tabular}

Instructions for use 


\title{
Misorientation/local plastic strain manifestations in chemical etching color
}

\author{
Xianguang Zhang ${ }^{1}$, Kiyotaka Matsuura ${ }^{2}$, Munekazu Ohno ${ }^{2}$ \\ 1Graduate School of Engineering, Hokkaido University, Kita 13 Nishi8, Kita-ku, Sapporo, Hokkaido \\ 060-8628, Japan
}

2 Division of Materials Science and Engineering, Faculty of Engineering, Hokkaido University, Kita 13 Nishi 8, Kita-ku, Sapporo, Hokkaido 060-8628, Japan

\begin{abstract}
:
The chemical etching contrast dependence of crystal orientation is investigated in both cold-deformed and undeformed iron. The chemical etching is performed by using nital, while the crystal orientation is determined by electron backscatter diffraction (EBSD). The results clearly show that the chemical etching contrast strongly depends on crystallographic orientation. The chemical etching contrast gives crystallographic information about the grain orientation and also the local orientation variations inside the individual grains due to plastic deformation. This provides a quite simple way to qualitatively understand the crystal orientation and misorientation in the microstructure.
\end{abstract}

Keywords: iron; metallography; chemical etching; crystal orientation; misorientation; electron backscatter diffraction (EBSD).

\section{Introduction}


Microstructures including crystal grains and constituent phases are made visible by etching. Two main types of etching are applied: the classical chemical etching and color etching. The chemical etching using an etchant is a very simple technique and is most widely used in the metallographic investigation, which removes the top layer of the metal surface in a selective way to reveal the microstructures. For example, ferrite grains of steel can be easily etched by nital and former austenite grain boundaries can be revealed by simple picric acid etching. On the other hand, so called color etching is carried out by deposition of a thin interface film on the top of the metal surface, causing light interference between the different reflections from the metal surface and the film surface (Voort, 1984).

It is known that the chemical etching is sensitive to the crystallographic orientation. Szabó and Bonyár (2012) investigated the effect of grain orientation on the chemical etching speed during pre-etching process of color etching, and they found that the etching speed strongly correlates with the grain orientation. The dependence of the etching rates of single-crystal silicon on crystallographic orientation has also been reported by Sato et al. (1999). Additionally, orientation-dependent topography formation was observed in FIB cutting (Lenius et al., 2011) and mechanical polishing (Zhu, 2004).

The orientation dependence of the color etching was reported by Ray et al. (1996) and Szabóet al. (2010), where the correlation between color shade and the crystal orientation was determined. However, according to their study, although color etchants allow distinguishing the different phases in most cases, the crystallographic 
orientation of the phases could not be detected, and the right etchants which can give the crystal orientation information needs to be tested (Szabóet al., 2010). Since the chemical etching is an easy way to reveal microstructures, if the correlation between the chemical etching-contrast and the crystal orientation also exists, it would provide a simpler way to understand the crystal orientation. In this regard, it is quite important to investigate the chemical etching contrast dependence of the crystal orientation. However, chemical etching has been used only for distinguishing the phases or grains from each other. The correlation between etching contrast and crystallographic orientation has not been seriously studied yet.

In this paper, the chemical etching contrast dependence of crystallographic orientation is studied. We focus on both the deformed and undeformed iron because the local crystallographic orientation inside the grain is changed due to plastic deformation. It will be shown in the present paper that various shades of chemical etching contrast give crystallographic information about both the grain orientation and orientation variations inside the individual grains.

\section{Experimental}

The material used in the present study is commercially pure Fe with a purity of 99.95 mass\% ( $\mathrm{Si}<0.01, \mathrm{Mn}<0.01, \mathrm{P}<0.002, \mathrm{~S}<0.002$ and $\mathrm{Al}<0.001$ mass\%.). The as-received pure Fe bars were annealed at $1273 \mathrm{~K}$ for $60 \mathrm{~min}$ and then slowly cooled to room temperature to obtain ferrite grains structure. The deformation was carried out by a ball-pressing (BP) test at room temperature; the set-up and procedure 
of which can be found in our previous report (Zhang et al., 2013). After the deformation, longitudinal mid-plane sections were prepared for electron backscatter diffraction (EBSD) measurement. The surface was polished by using $100 \mathrm{~nm}$ alumina polishing slurry followed by colloidal silica in order to achieve flat surfaces without any damage associated with the sample preparation for the EBSD analysis. That was an important step to obtain good diffraction patterns of the surface. The EBSD measurements were done with a JEOL JSM-6500F field-emission scanning electron microscope (FE-SEM) equipped with a TSL software. The step size was set to $3 \mu \mathrm{m}$ in all the cases. The grain boundary was defined as a boundary involving misorientation larger than $5^{\circ}$. The samples were chemically etched by $3 \%$ nital at room temperature for $5 \mathrm{sec}$. followed by optical microscopy (OM) observation. The difference in height of each grain on the etched surface was measured using an atomic force microscope (AFM; KEYENCE VN-8000) working in the tapping mode.

\section{Results and discussion}

Fig. 1(a) shows the grain structures of the undeformed sample after chemical etching taken by OM. It can be seen that the etching colors of the grains are various over the observation area. The etching color is unique to each grain and is, therefore, possibly related to their individual orientations. To confirm this, the same area of the sample was analyzed by EBSD, and the result is shown in Fig. 1(b). The individual grains are numbered in both the chemically etched image and the measured orientation map. By comparing Fig. 1(a) with (b), it can be clearly seen that different 
etching colors of the grains correspond to different grain orientations. Therefore, the variety of the etching-contrasts of the grains is a manifestation of variety of grain-orientations.
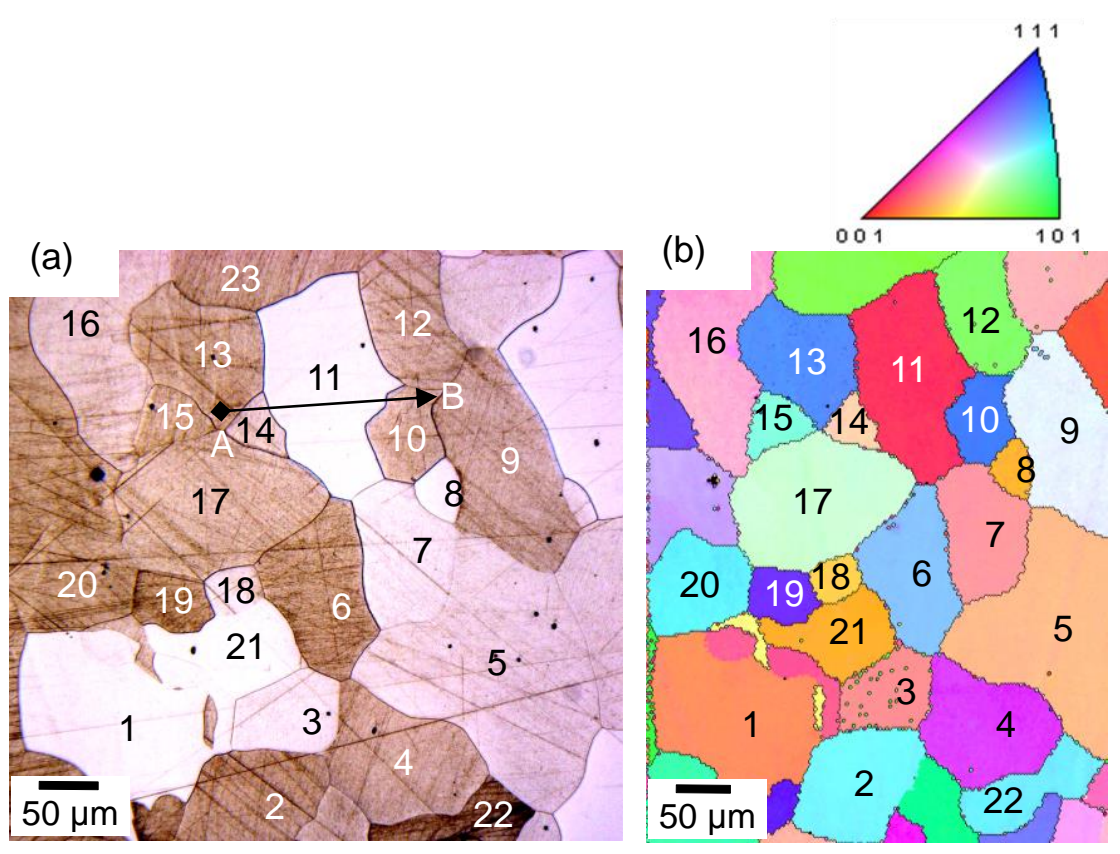

Fig. 1 (a) Optical image of chemical etching and (b) orientation map of the same sample area of the undeformed sample

It is very interesting to note that the gains exhibiting similar etched colors have similar orientations. For example, grains- 1, 3, 5, 7, 8, 11, 16, 18, and 21 in Fig. 1(a) exhibit relatively light color and Fig. 1(b) shows that they all have similar orientations close to [001]. On the other hand, the other grains exhibiting relatively dark color under OM have similar orientations close to [111] or [101].

Color components ( $R, G$ and $B$ ) (Where were they defined?) can be measured from Fig. 1(a) (How can they be measured?). The luminance $(Y)$ can be calculated by the following equation: [1] 
$Y=0.299 \cdot R+0.587 \cdot G+0.114 \cdot B$

The correlation between luminance and the angles between surface normal and [001], [101] and [111] directions is shown in Fig. 2. It can be clearly seen that the luminance nearly linearly decreases with the increasing angle $(\theta)$ between surface normal and [001] direction. By other words, when the grain orientation is getting close to [001] direction, the corresponding etching color tends to be brighter. While the grain orientation is getting close to [101] and [101] directions, the corresponding etching color tends to be darker. Hence, the grain orientations can be roughly identified from their chemically etched color, even though no apparent correlation exists between the luminance and the angle between surface normal and [111] and [101] directions, as shown in Fig. 2.

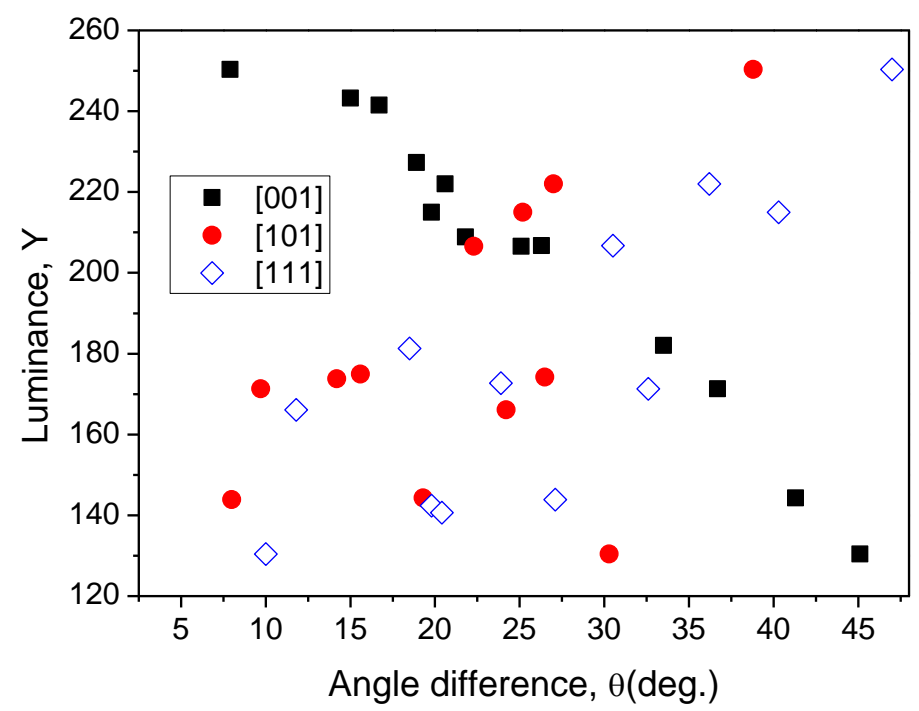

Fig. 2 Correlation between luminance and the angle between surface normal and [001], [101] and [111] directions. 
Fig. 3(a) shows the chemically etched image of the deformed sample and Fig. 3(b) is the corresponding crystal orientation map.

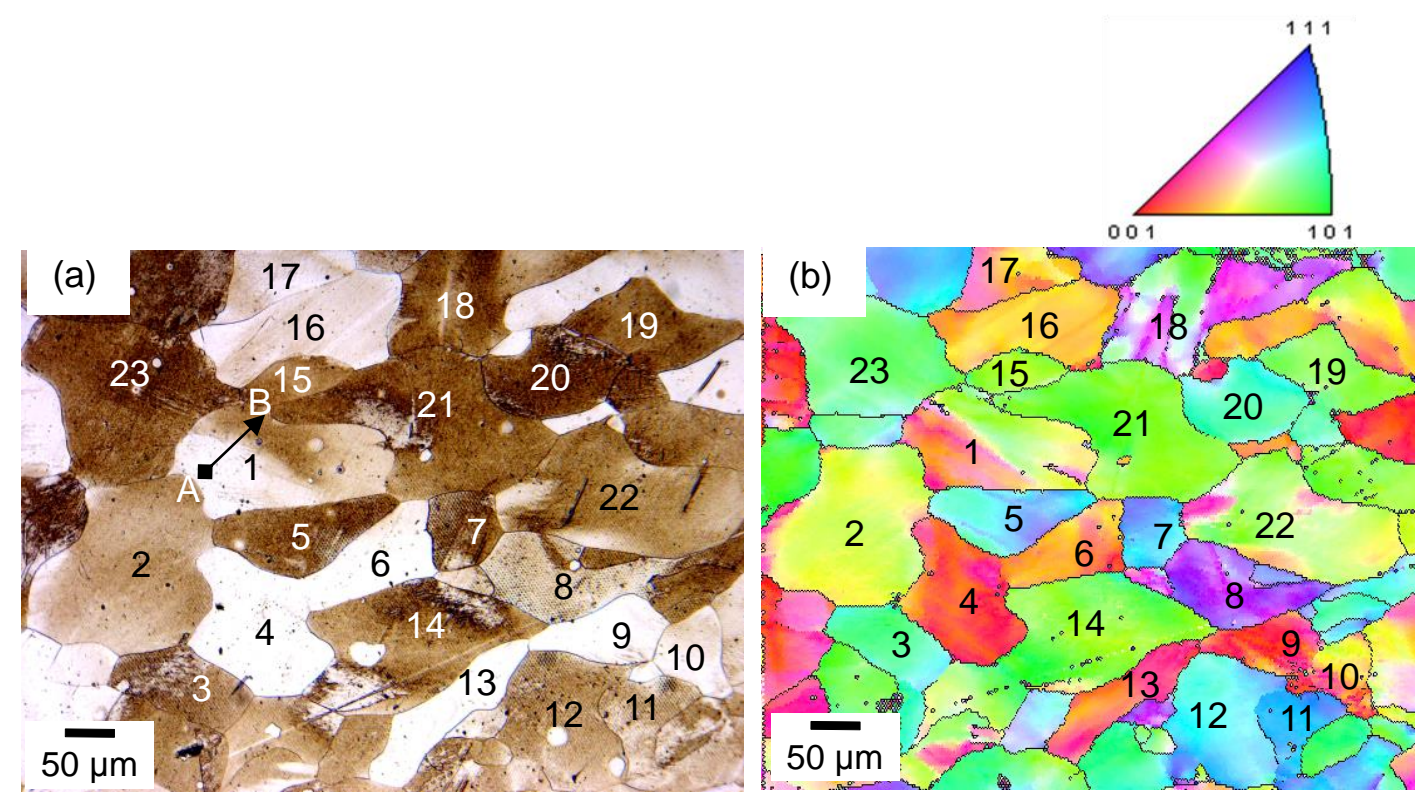

Fig. 3 (a) Optical image of chemical etching of the as-deformed sample and (b)

orientation map of the same sample area

From Fig. 3(b) it can be seen that the individual grains show varying shades of colors, indicating local rotation of crystal orientation inside the grain, due to deformation. This local change in the crystal orientation within the individual grain, defined as misorientation, has been used to evaluate the local strain (Zhang et al., 2013).

Importantly, gradual change of etching-colors inside the individual grains is observed in Fig. 3(a). By comparing Fig. 3(a) with Fig. 3(b), it can be noticed that this gradual change of etching-colors corresponds to the gradual change of crystallographic orientation inside the grain. For example, red and green colors in 
grain-1 in Fig. 3(a) indicating variation of crystallographic orientation, viz., the existence of strain inside the grain well correspond to the light and dark etching colors in the same grain in Fig. 3(b). The striking observation is that the subtle color-contrast displayed in grain-16 in Fig. 3(b) can even be finely revealed by the various shades of chemical etching-contrast in the same grain in Fig. 3(a). Hence, in the individual grains, when the local lattice orientation was changed by deformation, the corresponding etching contrast is also changed. The local lattice orientation variations, thus, the misorientations inside the grain can be revealed by the etching contrast. This provides a quite simple way to help understand the orientation variations inside the individual grains due to plastic deformation.

The average misorientation of grain $k, M_{k(\text { ave })}$ can be evaluated by the following equation (Zhang et al., 2013):

$$
M_{\mathrm{k}(\mathrm{ave})}=\sum_{i=1}^{n_{k}} f_{i, k} / n_{k}
$$

where $f_{i, k}$ denotes the misorientation angle at the point $i$ inside the grain $k$, which is quantified in reference to the average orientation of each grain, and $n_{k}$ is the number of points in the grain $k$.

By employing Eq. (2), the average misorientation of grains -1 and 22, were calculated to be $10.0^{\circ}$ and $5.8^{\circ}$, respectively, indicating that grain- 1 is more severely deformed. From Fig. 3(b) it can be seen that the etching contrast of grain-1 is stronger, while grain-22 is relatively lower, which corresponds to the varying degrees of misorientations, thus, the strain inside the grains. Therefore, the degrees of the 
misorientation of each grain can be qualitatively revealed by the varying levels of chemical etching-contrast. At the same time, dislocation densities and the degree of local strain which correspond to the degrees of misorientation of each grain can be also somehow qualitatively understood from the chemically etched image.

As is the same with the results of undeformed sample, the local region of the deformed grains with orientations near [001] also shows light etching-color, while orientations near [101] and [111] show dark etching color, indicating that the dependence of etching color on crystal orientation is reliable. This can be understood in Fig. 4, where the heights of the positions on the sample surface are plotted along the lines marked with $\mathrm{AB}$ in Figs. 1(a) and 3(a). It can be observed from line (a) in Fig. 4 that the surface height is different between grains. The grains with orientations near [001] exhibited higher surface height, while grains with orientations near [101] and [111] show lower grain-height. Grains with different height may give different light reflections, which results in various shades of etching colors under OM. It is very interesting that the surface of the individual deformed-grains also becomes irregular after chemical etching, as shown by line (b) in Fig. 4. The surface height gradually decreases from the chemically etched light-color region (orientations near [001]) to the dark-color region (orientations near [101] and [111]), which is consistent with the undeformed sample. The gradual change in surface height inside a grain leads to the various shades of etching-contrast taken by OM. 


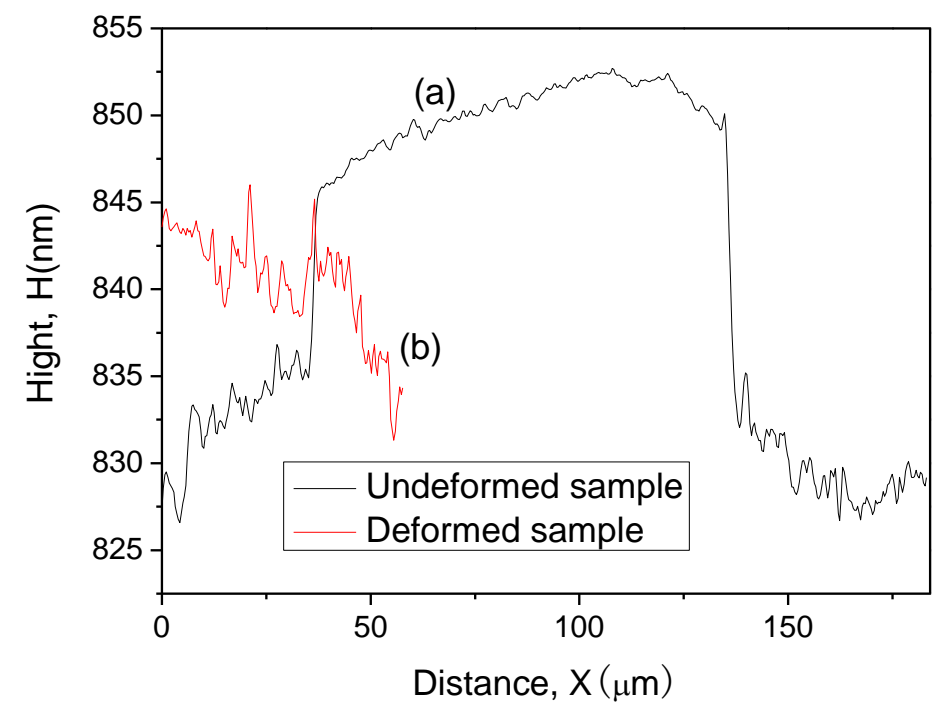

Fig. 4 The change of (a) grain height and (b) surface height inside a grain along the lines marked with $\mathrm{AB}$ presented in Figs. 1(a) and 3(a)

According to the above results, the chemical etching-contrast depends clearly on the crystallographic orientation of the grain. And the most striking fact here is the locally varying lattice orientations inside the individual grains can also be finely revealed by the etching contrast. Therefore, both the grain orientation and misorientation inside the individual deformed grains can be revealed by the chemical etching-contrast. This provides a direct and easy way to qualitatively understand the crystallographic orientation and misorientation in the microstructures.

\section{Conclusions}

Both cold-deformed and undeformed pure iron samples were chemically etched by using $3 \%$ nital etchant, while their crystal orientation was determined by 
electron backscatter diffraction (EBSD). It was found that the chemical etching-contrast clearly depends on the crystallographic orientation of the ferrite grain in iron. The crystallographic orientation dependent etching-contrast was found to give crystallographic information about both the grain orientation and misorientation inside the individual grains induced by the cold deformation. Therefore, the chemical etching can provide a direct and easy way to qualitatively understand the crystallographic orientation and misorientation in the microstructures.

\section{Acknowledgments}

This work was partially supported by "Nanotechnology Platform” Program of the Ministry of Education, Culture, Sports, Science and Technology (MEXT), Japan. One of the authors (X. Zhang) thanks Dr. S. Tsuchiya at Bridgestone Corporation for fruitful discussions.

\section{References}

A. Sáez-Maderuelo, L. Castro, G. de Diego: J. Nucl. Mater. 416 (2011), 75.

Lenius, M., Kree, R. \& Volkert, C.A. 2011!. Influence of crystal orientation on pattern formation of focused-ion-beam milled Cu surfaces. Phys Rev B 84, 035451.

M. Kamaya, A.J. Wilkinson, J.M. Titchmarsh: Acta Mater. 54(2006), 539.

M. Kamaya, A.J. Wilkinson, J.M. Titchmarsh: Nucl. Eng. Des. 235(2005), 713.

R. C. Gifkens, The polarizing microscope, in Optical Microscopy of Metals, Sir Issac Pitman and Sons Limited, Melbourne, p. 103-123 (1970). 
Ray, A. \& Dhua, S.K. 1996!. Microstructural manifestations in color: Some applications for steels. Mater Charact 37, 1-8.

Szabó, P.J. \& Bonyár, A. 2012!. Effect of grain orientation on chemical etching. Micron 43, 349-351.

Szabó, P.J. \& Kardos, I. 2010!. Correlation between grain orientation and the shade of color etching. Mater Charact 61, 814-817.

Sato, K., Shikida, M., Yamashiro, T., Asaumi, K., Iriye, Y., Yamamoto, M. (1999). Anisotropic etching rates of single-crystal silicon for TMAH water solution as a function of crystallographic orientation. Sensors and Actuators A: Physical, 73(1), $131-137$.

Ueda, T., Kohsaka, F., Iino, T. \& Yamazaki, D. 1987!. Theory to predict etching shapes in quartz and application to design devices. T Soc Instrum Control Eng 23, $1233-1238$.

Voort, G.V. 1984!. Metallography. Principles and Practice. New York:

McGraw-Hill Book Co. and Materials Park, OH: ASM International.

X. Zhang, K. Matsuura, M. Ohno, S. Suzuki: Mater. Sci. Eng. A, 564(2013), 169.

Zhu, H. 2004!. Chemical mechanical polishing CMP! Anisotropy in sapphire. Appl Surf Sci 236, 120-130.

5] Cheng HD. Color image segmentation: advances and prospects. Pattern Recognition 2001;34:2259-81. 